EPJ Web of Conferences 42, 06005 (2013)

DOI: $10.1051 /$ epjconf/20134206005

(C) Owned by the authors, published by EDP Sciences, 2013

\title{
Modelling the widths of fission observables in GEF
}

\author{
B. Jurado ${ }^{1}$, K.-H. Schmidt ${ }^{1}$ \\ ${ }^{1}$ CENBG, CNRS/IN2P3, 33175 Gradignan, France
}

\begin{abstract}
The widths of the mass distributions of the different fission channels are traced back to the probability distributions of the corresponding quantum oscillators that are coupled to the heat bath, which is formed by the intrinsic degrees of freedom of the fissioning system under the influence of pairing correlations and shell effects. Following conclusion from stochastic calculations of Adeev and Pashkevich, an early freezing due to dynamical effects is assumed. It is shown that the mass width of the fission channels in low-energy fission is strongly influenced by the zero-point motion of the corresponding quantum oscillator. The observed variation of the mass widths of the asymmetric fission channels with excitation energy is attributed to the energy-dependent properties of the heat bath and not to the population of excited states of the corresponding quantum oscillator.
\end{abstract}

\section{Introduction}

The GEneral Fission code GEF [1] calculates a variety of observables such as fission-fragment yields, prompt-fission neutrons and gammas. Contrary to most fission models, GEF can be used to predict these quantities for fissioning nuclei for which no experimental data are available. In this contribution we present a modified application of the statistical model that is used in GEF for estimating the widths of the mass distributions of the fission channels.

\section{Dynamical effects}

There is a long tradition in applying the statistical model to nuclear fission [2-5]. However, it is well known [6] that the statistical model, applied to the scission-point configuration, is unable of explaining the variances of the mass and energy distributions and their dependence on the compound-nucleus fissility parameter. The solution of the problem has been given by Adeev and Pashkevich [7] by considering the influence of inertia and dissipation. Their calculations suggest that the width of the distribution of a specific normal mode is approximately given by the fluctuation of the corresponding oscillator with an effective stiffness that is equal to the stiffness of the potential somewhere between saddle and scission. Thus, the application of the statistical model to fission seems to be a reasonable approximation. However, it is important to consider dynamical effects by assuming that the collective coordinate is frozen somewhere before scission. The configuration, where the statistical model is to be applied is specific to the collective coordinate considered.

\section{Quantum oscillator}

The potential $U$ in the vicinity of a minimum as a function of a collective coordinate $q$ is approximated by a parabola

This is an Open Access article distributed under the terms of the Creative Commons Attribution License 2.0, which permits unrestricted use, distribution, and reproduction in any medium, provided the original work is properly cited. 


$$
U=\frac{1}{2} C q^{2}
$$

Thus, the motion along the collective coordinate $q$ corresponds to an excited state of an harmonic quantum oscillator. In an excited nucleus, there is an exchange of energy between the specific collective and all the other nuclear degrees of freedom that may be considered as a heat bath. In thermal equilibrium, the properties of the heat bath (e.g. state density and temperature $T$ ) determine the probability distribution of excited states of the harmonic oscillator considered. From this distribution, one can derive the probability distribution to find the oscillator at a specific coordinate value $q$ at a given time.

Some analytical solutions to this problem for specific cases are well known [8,9]: If the excitation energy is zero, the probability distribution $P(q)$ along coordinate $q$ of an harmonic quantum oscillator with a characteristic energy $\hbar \omega$ has the shape of a Gaussian function with the variance

$$
\sigma_{q}^{2}=\frac{\hbar \omega}{2 C}
$$

as given by the zero-point motion.

For finite excitation energies, the probability distribution along the coordinate $q$ is the sum of the contributions from different excited states of the oscillator

$$
P(q)=\sum_{0}^{i_{\max }} W_{i} \cdot\left|\varphi_{i}(q)\right|^{2}
$$

where $W_{\mathrm{i}}$ is the population probability of the state $i$ of the oscillator with excitation energy $E_{\mathrm{i}}=i \cdot \hbar \omega$, $\varphi_{i}(q)$ is the wave function of this state, and $i_{\max }$ is the largest integer which fulfils the condition $i_{\max } \leq \frac{E_{t o t}}{\hbar \omega}$.

If the heat bath is described by the constant-temperature level density, then the population probabilities $W_{\mathrm{i}}$ are given by a Boltzmann distribution:

$$
W_{i} \propto \exp \left(-E_{i} / T\right)
$$

In that case if $E_{t o t}=\infty$, the probability distribution $P(q)$ has a Gaussian shape, and the variance is given by the following expression:

$$
\sigma_{q}^{2}=\frac{\hbar \omega}{2 C} \operatorname{coth}\left(\frac{\hbar \omega}{2 T}\right)
$$

This relation approximately also holds if $E_{t o t}>T$.

If in addition $T>>\hbar \omega$, the variance of the probability distribution $P(q)$ approaches the classical value:

$$
\sigma_{q}^{2}=T / C
$$

\section{Experimental data}

\subsection{Symmetric fission channel}

The mass width of the symmetric fission channel and its dependence on excitation energy have been investigated in a broad range of fissioning nuclei, from $Z^{2} / A=28$ to $Z^{2} / A=43[10,11]$. 
First measurements of the symmetric fission channel at low excitation energy have been performed with radioactive targets around ${ }^{226} \mathrm{Ra}[12,13]$. More recently, systematic measurements of electromagnetic-induced fission of ${ }^{238} \mathrm{U}$ projectile fragments have been performed on a larger number of nuclei in this region [14]. This experiment provided precise data on the standard deviation of the $Z$ distribution of the symmetric fission channel. A value of $\sigma_{Z}=4$ units was found, which is nearly constant within the experimental uncertainties in the range from ${ }^{212} \mathrm{Ac}$ to ${ }^{226} \mathrm{Th}$ for excitation energies of a few $\mathrm{MeV}$ above the fission barrier. From this value one can deduce a value of $\sigma_{A}=10$ mass units for the mass width of the symmetric channel $[14,15]$. There are no experimental data available on the evolution of the width with excitation energy for the symmetric fission channel in low-energy fission.

\subsection{Asymmetric fission channels}

The mass distributions of the asymmetric fission channels, Standard 1 and Standard 2, which are distributed to shell effects, are much narrower: $\sigma_{A}$ is around 3, respectively 5 mass units [15]. The widths increase slightly with growing initial excitation energy of the fissioning system. This is clearly visible by varying the neutron energy in neutron-induced fission (see work cited in [16] and work cited in [17-19]). Fig. 1 shows this increase for neutron-induced fission of ${ }^{237} \mathrm{~Np}$ [19].

\section{Theoretical description}

\subsection{Symmetric fission channel}

The evolution of the width of the mass distribution of the symmetric fission channel at higher excitation energies, where shell effects are essentially washed out, has been the subject of longstanding experimental and theoretical investigations, see refs. [20-23]. The main results are:

- The width of the mass distribution is well described by the analytical approximation $\sigma_{q}=\sqrt{\frac{\sqrt{E_{t o t} / a}}{C}}$ that corresponds to equation (6) with the temperature defined by the Fermi-gas nuclear level density.

- The corresponding stiffness $C$ of the mass-asymmetric potential (e.g. $C=0.0049 \mathrm{MeV}$ for $\left.{ }^{238} \mathrm{~Np}[10]\right)$ is in agreement with theoretical estimates for a configuration close to saddle [24].

Stimulated by the success in describing the symmetric fission channel at higher excitation energy, we extend the statistical approach to lower energies. Application of equation (5) with $\mathrm{C}=0.0049$ $\mathrm{MeV}, T=0.45 \mathrm{MeV}$ [25], and $\sigma_{A}=10$ units yields $\hbar \omega=0.5 \mathrm{MeV}$. Under these conditions ( $\hbar \omega \approx T$ ) the mass width of the symmetric channel in low-energy fission of light actinides is strongly influenced by the zero-point motion. In comparison, Nix estimated a value of $\hbar \omega$ for ${ }^{238} \mathrm{~Np}$ in the order of 1 to $2 \mathrm{MeV}$ in the actinide region $\left(\hbar \omega=1.2 \mathrm{MeV}\right.$ for $\left.{ }^{238} \mathrm{~Np}\right)$ for mass-asymmetric shape distortions at the saddle-point [26].

\subsection{Asymmetric fission channels}

Assuming the inertia to be the same as for the symmetric fission channel, taking the experimental widths at the lowest values of $E_{n}$ from Fig. 1 and the temperature of the heat bath from the empirical constant-temperature level density systematics of ref. [25], eq. (2) gives $\hbar \omega=3.3 \mathrm{MeV}$ for S2 and $\hbar \omega=8.9 \mathrm{MeV}$ for $\mathrm{S} 1$. Note that eq. (2) can be used, because $T<<\hbar \omega$. Besides, we consider (in accordance with ref. [24]) that the mass distribution is determined close to the outer saddle point. Thus, the excitation energy of the heat bath is assumed to be equal to the nuclear excitation energy 
above the outer saddle. In the energy regime covered by the data shown in Fig. 1, the population of the first excited state of the oscillator is not possible for the $\mathrm{S} 1$ fission channel, since $E_{t o t}<\hbar \omega$, and negligible for the S2 fission channel, since $T<<\hbar \omega$. Thus, the widths of the mass distributions of the asymmetric fission channels are determined by the width of the zero-point motion, only. The deduced value of $\hbar \omega=3.3 \mathrm{MeV}$ for the S2 fission channel can be compared with an estimation of Mirea with the microscopic-macroscopic model using the cranking method who obtained $\hbar \omega=7.26$ $\mathrm{MeV}$ at the outer saddle and $\hbar \omega=3.76 \mathrm{MeV}$ at a distance between the centers of the nascent fragments of $1 \mathrm{fm}$ behind the outer saddle [27]. Thus, the deduced empirical value of $\hbar \omega$ is half the theoretical value at the barrier, like in the case of the symmetric fission channel.

It is a crucial test of our assumptions to verify whether the increase of the mass widths of the asymmetric fission channels with increasing initial excitation energy (see Fig. 1) can be explained by the decrease of the effective stiffness due to the washing out of the shell effect in the level density. The restoring force $F(q)$ of an oscillator that is coupled to a heat bath is given by

$$
F(q)=T \frac{d S}{d q}
$$

The potential is given by integration: $U(q)=\int F(q) d q$, and the effective stiffness by the second derivative of the potential: $C_{\text {eff }}=d^{2} U / d q^{2}$. The effective stiffness $C_{\text {eff }}$ in the vicinity of the potential minimum was determined numerically for the oscillator potential of the corresponding asymmetric fission channel. The temperature $T(q)$ and the entropy $S=\ln (\rho)=E / T$ are defined by the equations

$$
\rho \propto \frac{1}{T} \exp (E / T)
$$

where $\mathrm{T}$ is obtained from the parameterization of ref. [25]:

$$
T=\frac{1}{A^{2 / 3}}\left[17.45-0.51 \cdot \delta U+0.051 \cdot(\delta U)^{2}\right]
$$

In this case it is important to consider the shell effect $\delta U$ that forms the respective fission valley:

$$
\delta U(q)=(\delta U)_{0}+\frac{C}{2}\left(q-q_{0}\right)^{2}
$$

A value of $(\delta U)_{0}=-5 \mathrm{MeV}$ was assumed from shell-model calculations [28,29] as a rough estimate for both channels.

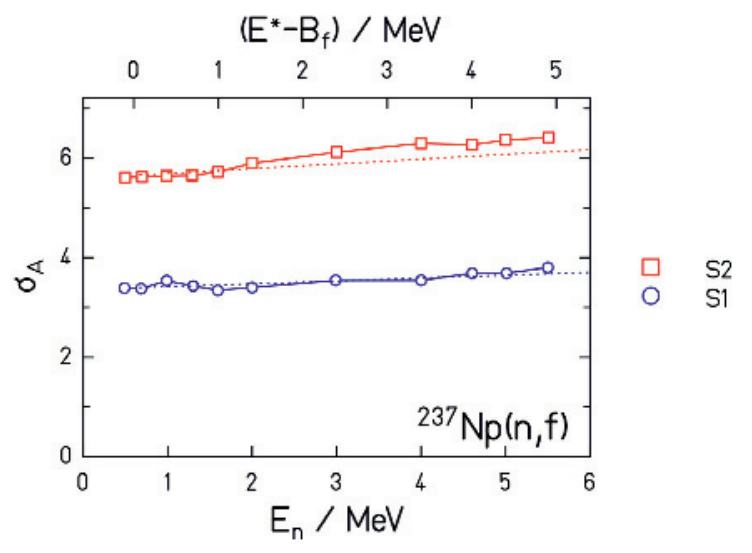

Fig. 1: Standard deviation of the mass distribution of the asymmetric fission channels (Standard 1 and Standard 2) in the fission of ${ }^{237} \mathrm{~Np}(\mathrm{n}, \mathrm{f})$ as a function of the neutron energy $E_{n}$ (lower scale) and the excitation energy above the outer fission barrier $B_{f}$ (upper scale). $B_{f}=6.1 \mathrm{MeV}$ is taken from ref. [30]. The measured data [19] (symbols) are compared to the widths of the corresponding quantum oscillators (dotted lines). 
Assuming a constant inertia, the variation of the width of the zero-point motion of the corresponding quantum oscillator is given by the variation of the effective stiffness $C_{\text {eff }}: \sigma_{A}^{2} \propto \sqrt{1 / C_{\text {eff }}}$. The result of this estimation is shown in figure 1 by the dotted lines. It agrees rather well with the data. Thus, the quantum oscillator with the energy-dependent stiffness, once adjusted to the measured width at zero excitation energy above the outer saddle, also reproduces the observed growth of the mass width $\sigma_{\mathrm{A}}$ of the asymmetric fission channels up to $E_{\text {tot }}-B_{f}=5 \mathrm{MeV}$, where experimental data are available.

\section{Conclusion}

One may conclude that several features of low-energy fission can successfully be described on the basis of the statistical model provided that dynamical effects, the finite total energy and the quantummechanical properties of the system are properly considered. A specific, important result of this work is the evidence that the mass distributions of the fission channels in low-energy fission are strongly influenced or even essentially given by the zero-point motion of the quantum oscillators in the corresponding fission valleys. This implies that an approach which considers the quantummechanical properties of the system is mandatory for a realistic estimation of the width of the mass distributions of the different fission channels in low-energy fission.

\section{Acknowledgement}

This work was supported by the European Commission within the Seventh Framework Programme through Fission-2010-ERINDA (project no.269499).

\section{References}

1. K.-H. Schmidt, B. Jurado, JEF/DOC 1423, OECD Nuclear-Energy Agency, Paris (2012)

2. P. Fong, Statistical Theory of Nuclear Fission (Gordon and Breach, New York, 1969)

3. G. A. Pik-Pichak and V. M. Strutinsky, Physics of the fission of the atomic nuclei, eds. N. A.

Perfilov and V. P. Eismont, (Moscow, Gosatmomizdat, 1962) p. 12

4. A. V. Ignatyuk, Yad. Fiz. 9, 357 (1969)

5. B. D. Wilkins, E. P. Steinberg, R. R. Chasman, Phys. Rev. C 14, 1832 (1976)

6. Yu. Ts. Oganessian and Yu. A. Lazarev, Treatise on Heavy Ion Science. Vol. 4, ed. D. A.

Bromley, (Plenum Press, New York, 1985) p. 1.

7. G. D. Adeev, V. V. Pashkevich, Nucl. Phys. A 502, 405c (1989)

8. L. D. Landau and E. M. Lifshitz, Statistical physics, translated by E. Peierls and R. F. Peierls

(Addison-Wesley Publ. Co., Reading, Mass., 1958) pp. 86-89

9. J. R. Nix, Nucl. Phys. A 130, 241 (1969)

10. A. Ya. Rusanov, M. G. 1tkis, V. N. Okolovich, Phys. At. Nucl. 60, 683 (1997)

11. S. I. Mulgin, K.-H. Schmidt, A. Grewe, S. V. Zhdanov, Nucl. Phys. A 640, 375 (1998)

12. E. Konecny, H. J. Specht, J. Weber, Phys. Lett. B 45, 329 (1973)

13. E. A. Zhagrov et al., Nucl. Phys. A 213, 436 (1973)

14. K.-H. Schmidt et al., Nucl. Phys. A 665, 221 (2000)

15. C. Böckstiegel et al, Nucl. Phys. A 802, 12 (2008)

16. T.-s. Fan, J.-m. Hu, S.-1. Bao, Nucl. Phys. A 591, 16 (1995)

17. U. Brosa, H.-H. Knitter, T.-s. Fan, J.-m. Hu, S.-1. Bao, Phys. Rev. C 59, 767 (1999)

18. F. Vivès, F.-J. Hambsch, H. Bax, S. Oberstedt, Nucl. Phys. A 662, 63 (2000)

19. F.-J. Hambsch, F. Vivès, P. Siegler, S. Oberstedt, Nucl. Phys. A 679, 3 (2000)

20. J. R. Nix, W. J. Swiatecki, Nucl. Phys. 71, 1 (1965)

21. G. D. Adeev, V. V. Pashkevich, Nucl. Phys. A 502, 405c (1989) 
22. M. G. Itkis et al., Phys. At. Nucl. 58, 2026 (1995)

23. A. Ya. Rusanov, V. V. Pashkevich, M. G. Itkis, Phys. At. Nucl. 62, 547 (1999)

24. E. G. Ryabov et al., Phys. Rev. C 78, 044614 (2008)

25. T. von Egidy, D. Bucurescu, Phys. Rev. C 72, 044311 (2005)

26. J. R. Nix, Ann. Phys. 41, 52 (1967)

27. M. Mirea, private communication, 2012

28. B. D. Wilkins, E. P. Steinberg, R. R. Chasman, Phys. Rev. C 14, 1832 (1976)

29. I. Ragnarsson, R. K. Sheline, Phys. Scr. 29, 385 (1984)

30. S. Bjørnholm, J. E. Lynn, Rev. Mod. Phys. 52, 725 (1980) 